\title{
OFFLINE SIGNATURE VERIFICATION SYSTEM FOR BANK CHEQUES USING ZERNIKE MOMENTS, CIRCULARITY PROPERTY AND NEURAL NETWORK
}

\author{
Ashok Kumar. $\mathrm{D}^{1}$, Dhandapani. $\mathrm{S}^{2}$ \\ ${ }^{1}$ Department of Computer Science and Applications Government Arts College, \\ Tiruchirapalli - 620022 \\ ${ }^{2}$ Department of Computer Science and Applications Government Arts College, \\ Tiruchirapalli - 620022
}

\begin{abstract}
Handwritten signature is the most accepted and economical means of personnel authentication. It can be verified using online or offline schemes. This paper proposes a signature verification model by combining Zernike moments feature with circularity and aspect ratio. Unlike characters, signatures vary each time because of its behavioural biometric property. Signatures can be identified based on their shape. Moments are the good translational and scale invariant shape descriptors. The amplitude and the phase of Zernike moments, circularity and aspect ratio of the signature are the features that are extracted and combined for the verification purpose and are fed to the Feedforward Backpropagation Neural Network. This Neural Network classifies the signature into genuine or forged. Experimental results reveal that this methodology of combining zernike moments along with the two mentioned geometrical properties give higher accuracy than using them individually. The combination of these feature vector yields a mean accuracy of $95.83 \%$. When this approach is compared with the literature, it proves to be more effective.
\end{abstract}

\section{KEYWORDS:}

Offline Signature Verification, Zernike Moments, Neural Network.

\section{INTRODUCTION}

Signature is the widely used and accepted form of authentication and it is used even before the usage of computers [1]. It is easier because it does not require any physical hardware to capture as that of fingerprints. Signature takes the advantage of traditional biometric methods like passwords, PIN and ID cards which can be stolen, lost or one may forget. Handwritten signatures are used in almost all documents where authentication is required. It has low conflict percentage. The number of bank cheque fraud cases are rising and there are number of modern techniques followed by the fraudulent to counterfeit. The cheque is washed with special fluid to erase the 
amount figures and writing a huge amount, changing the name of the bearer, removing the signature and placing duplicated signatures are the different ways cheque fraud is reported. To avoid such offense activities, a model to verify signature is badly needed. Reserve Bank of India introduced cheque truncation system which will minimize the above problems [2]. Signatures are not easy to verify like that of characters. Verifying signatures in bank cheques is a challenging opportunity in image processing. Characters have definite shape and can easily be identified. Since signature is a behavioral biometric, it varies according to the mood, environmental condition and presence of mind of the signer. No one can put the signature alike all the times. There may be small variations which are called intra-class variations. These variations are to be neglected during the verification process. Only inter-class variations verify and classify the signatures into genuine or forged. Handwritten signatures can be verified using online or offline schemes. Online signature can be captured using electronic devices like writing pad or stylus attached to a computer. It can capture dynamic features, like writing speed, angle, number of pen ups and time taken to put the signature, which will make the verification process easier and more accurate. Offline signature will have only the digitised signature from which required features can be extracted. Since this paper deals with signatures on bank cheques, offline signature verification scheme is chosen. A signature which is suspicious is called a forged signature. Forgery can be of four types. Simple forgery is the forger writing the name of the signer casually. Random forgery is the forger trying to imitate the genuine signature. Skilled forgery is the forger trying to put the near exact signature by many practices. Disguised forgery is the forgery that the genuine user himself puts his signature incorrectly to later deny that he has not signed. The proposed model uses CEDAR (Center of Excellence of Document Analysis and Recognition) database which is publicly available. Neural Network is used for the classification purpose even though it has some limitations [3]. Signature verification is a two-class pattern recognition problem. The output of the neural network may be ' 0 ' or ' 1 '. Feedforward neural network is used for the verification purpose. The neural network is trained using the Backpropagation algorithm.

The breakdown of this paper is as follows. Section 2 deals with Literature Review. Section 3 deals with image acquisition and preprocessing works done for the signature. Section 4 describes the feature extraction part. Section 5 describes the Neural Network and Classification. Section 6 describes the experimental results and discussion and Section 7 finally concludes the research paper.

\section{LITERATURE REVIEW}

In [4], Rajesh kumar etal proposed a signature verification system using the surroundedness feature and CEDAR database and achieved $91.67 \%$ of accuracy. Hai-Lin and Hai-zhou [5] proposed a verification method using Zernike moments since it is rotation and scale invariant. Pushpalatha etal [6] proposed a verification method with polar feature descriptor for signature that contains radon transformation and zernike moments. The system detects skilled forgery with an accuracy of $71 \%$. Khotanzad and Hong [7] studied the superiority of Zernike moment feature over regular moments and moments invariants. Sohail and Rashid [8] combined circularity with other structural features and have gained good results. Jovisa [9], defined the circularity measure which is robust to noise and it is invariant to translation, rotation and scaling. Huazhong etal [10] presented a brief description of types of moments and reveal that Zernike moments are invariant 
to rotation transformation. Divjyot and Dutta [11] used Zernike moments and minutae features for signature verification. The experimental results show that Zernike moments excel with an accuracy rate of $95.55 \%$ when compared to minutae feature with an accuracy of $95.22 \%$. Neo Han etal [12] combined a new feature set using Zernike moments and wavelet transforms and got an accuracy rate of $94.26 \%$ for face recognition which is tabulated in Table I for comparative analysis with the proposed methodology. Pham etal [13] extracted features using an adaptation of the shape context descriptor which were robust to noise, rotation and scaling. This lead them not to depend upon the preprocessing activities. Cemil Oz [14] proposed a signature verification method with two separate sequential neural networks, one for recognition and other for verification. He used moment invariant methods for feature extraction and found that the performance was good enough to verify the signature. Pradeep and etal [15] proposed a signature verification system using the shape descriptor and Euclidian distance. They used correspondences between signatures, aligned transforms and got encouraging results.

\section{IMAGE ACQUISITION AND PREPROCESSING}

The process of converting the hardcopy of the bank cheque into soft copy for digitization of the image is called image acquisition. It can be done in two ways. The signature can photographed or scanned using a high resolution scanner. Signature verification systems normally face the problem of number of signature samples available for training the classifier. In real, banks mostly get three specimen signature samples. Those samples are taken immediately and so the customer signs casually one after another three times. Intra personnel variations can be recorded if the signature samples are collected at different times. Neural Network requires an adequate amount of samples for training which will not be practically possible. Diaz and etal [16] have proposed an idea to create duplicate signatures using a cognitive inspired algorithm. It is based on transformations which simulate the human spatial cognitive map and motor system intra-personal variability during the signing process.

Preprocessing is done to remove the noise from the image. The noise may have crept during scanning or folding of cheque leaves. Bank cheque usually contains logo and some background images or patterns. The signature may be on the background part and that has to be removed. Only the signature part alone is to be isolated. A threshold is used to make other pixels white and the signature pixels black. Preprocessing improves accuracy and classification rate. The normal steps in preprocessing are converting to grayscale, binarisation, segmentation. Median filters can be used to remove external noise [17]. Processing images in gray scale is easier than RGB. Binarisation will have only 0 or 1 pixels instead of 256 gray levels which may make the task little complex. Area of interest, signature portion, is located by segmentation process. Normalization is done for making the verification process easy and to extract feature values accurately [17].

\section{FEATURE EXTRACTION}

Feature extraction is the vital portion of signature verification process. It is the process of defining image characteristics to represent the image information more meaningfully and efficiently. The effectiveness of feature extraction can be increased by reducing number of features used and maximizing pattern discrimination [18]. It is used for further analysis and 
International Journal of Artificial Intelligence and Applications (IJAIA), Vol. 7, No. 5, September 2016

classification purposes. Aspect Ratio is the ratio of the number of columns to the number of rows. The circularity of the signature is calculated with its Perimeter (p) and Area (Ar) by

$$
\text { Circularity }=\frac{4 \pi A r}{P^{2}} \quad-
$$

Moments are the count of number of objects at a distance from a point. They are simple. Translational and scale invariant shape descriptors [19]. Moment descriptors has the high quality in representing shapes [20]. Zernike moments are chosen because of its robustness towards image noise, geometrical invariance property and orthogonal property. Using Zernike moments reduces the false negative rate [21]. Algorithm I is used to extract the moment features.

Zernike Polynomials are an orthogonal set of complex-valued polynomials represented as

$$
\begin{aligned}
& \operatorname{Vnm}(x, y)=R_{n m}(x, y) \cdot \exp \left(\left(j m \cdot \tan ^{-1} \frac{y}{x}\right)\right) \\
& \text { where, } \\
& \qquad x^{2}+y^{2} \leq 1, j=\sqrt{-1}, n \geq 0,|m| \leq n
\end{aligned}
$$

and $\quad n-|m|$ is even and Radial polynomials $\left\{R_{n m}\right\}$ are defined as

$$
\begin{aligned}
& R_{n, m}(x, y)=\sum_{s=0}^{\frac{n-|m|}{2}} b_{n|m| s}\left(x^{2}+y^{2}\right)^{\frac{n}{2}-s} \\
& \text { where, } \\
& \qquad B_{n}|m| s=\frac{(-1)^{s}(s-1) !}{s !\left(\frac{n+|m|}{2}-s\right) !\left(\frac{n+|m|}{2}-s\right) !}
\end{aligned}
$$

The complex Zernike moments of order $\mathrm{n}$ and repetition $\mathrm{m}$ are given by

$$
\begin{aligned}
& A_{n m}=\frac{n+1}{\pi} \sum_{k} \sum_{y} f(x, y) V_{n m}^{*}(x, y) \\
& \text { where, } \\
& \qquad x^{2}+y^{2} \leq 1 \text { and symbol } * \text { denotes the complex conjugate operator. }
\end{aligned}
$$

The Zernike moments can be computed using the scale invariant central moments by 
International Journal of Artificial Intelligence and Applications (IJAIA), Vol. 7, No. 5, September 2016

$$
\frac{n+1}{n} \sum_{\frac{k-|m|}{n-k=\text { even }}}^{n} \sum_{a=0}^{b} \sum_{d=0}^{|m|}\left(-j^{d}\right) \cdot\left(\left|\frac{m}{d}\right|\right)\left(\frac{b}{a}\right) B_{n|m| s} G_{k-2 a-d, 2 a+d} \quad-
$$

where

$$
b=\frac{n-|m|}{2}-S \text { and } j=\sqrt{-1}
$$

The meshgrid function in Algorithm I replicates the grid vectors $\mathrm{x}$ and $\mathrm{y}$ to produce full grid by $\mathrm{X}$ and Y. The signature's orientation angle can be found using the phase angle of the Zernike moments. The order of the Zernike moments is set to 4 and the repetition number of the Zernike moments is set to 2 .

Algorithm I: Extraction of Amplitude and Phase Angle from Moments

\section{Input :}

$P$, input image matrix, $n$, order of the Zernike moments, $m$, repetition number of Zernike moments.

\section{Output :}

A, Amplitutde of the moment and Phi, the phase (angle) of the moment.

\section{Method:}

Procedure calculatezm $(\mathrm{p}, \mathrm{nm})$

begin

$\mathrm{N} \leftarrow \operatorname{size}(\mathrm{p}) ;$

begin $\mathrm{x} \leftarrow 1$ to $\mathrm{N}, \mathrm{y} \leftarrow \mathrm{x}$;

end

$$
\mathrm{XY} \leftarrow \operatorname{meshgrid}(\mathrm{x}, \mathrm{y})
$$

calculate the radial polynomial using the equation (3)

calculate moments by using the equation (4)

end

calculate amplitude $(\mathrm{A})$ and phase angle(Phi) from the moments.

The radial polynomial is first obtained using equation (3) and the moments are calculated using equation (5). Equation (2) gives the zernike polynomials from which amplitude and phase angle are calculated.

\section{CLASSIFICATION WITH NEURAL NETWORK}

Artificial Neural Networks (ANN) is acquiring significant importance for pattern recognition applications particularly in the field of biometrics. Neural networks have got a renaissance because of the highly super computing power the industry has advanced. Neural network is a 
function which can mimic the learning process of humans. This catches attraction over it. ANN is accepted widely because of its learning ability, fault tolerant, robustness, learning by example and generalizing ability. Outputs, produced by the neural network, depend on model parameter values for each network layer [22]. They don't depend on input data and labels. ANN can handle non-convex decisions [23]. Several computational techniques are available in the literature for classification but Artificial Neural Network (ANN) has outperformed other automated techniques because of the various advantages such as high accuracy and quick convergence. Neural network is defined by its architecture, neuron model, activation function and the learning algorithm.

The neural network modeled for the signature verification has 4 inputs. Amplitude and phase angle of Zernike moments, circularity and aspect ratio are the inputs and the network uses feedforward backpropagation architecture. There is one input layer, one hidden layer and an output layer. The number of neurons is varied iteratively and fixed as 10 in the hidden layer. The weights (W) and bias (b) are initialized randomly and the output values decide whether the signature given for test is genuine or forged. Figure 2 shows the neuron designed for the signature verification process. The activation function used is sigmoid since it can deliver the output values from 0 to 1 . The feedforward backpropagation neural network architecture designed is first trained by the sample signatures, validated and tested for signatures.

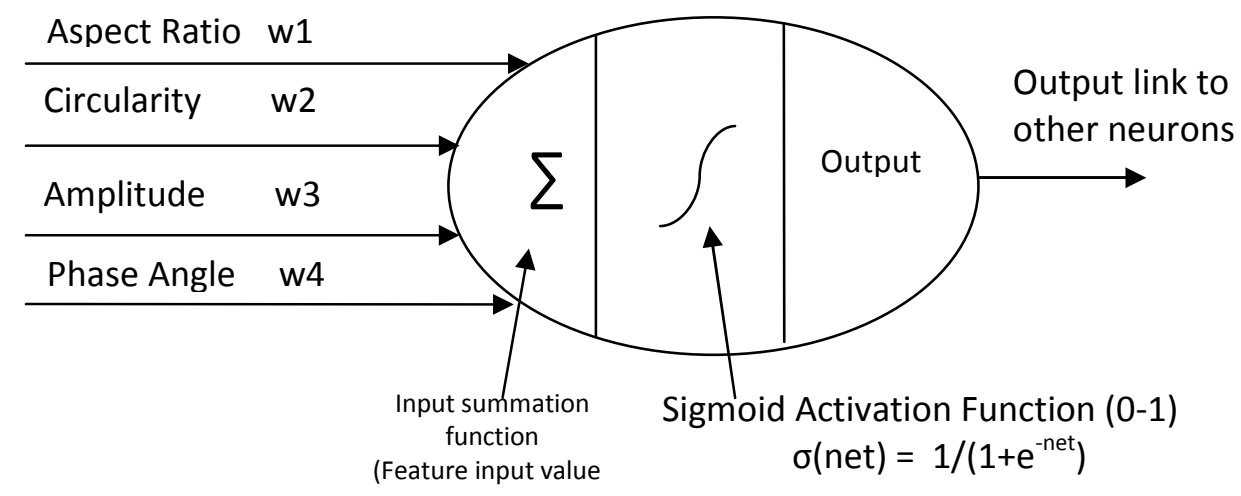

Figure.2: A Neuron designed for signature verification

Algorithm II gives the basic steps of feedforward backpropagation neural network. The weights and bias are initialized to random values. Sigmoid is used for activation function because it tunes the output from 0 to 1 . Here 0 represents forged signature and a 1 represents genuine signature. The activation function is applied on the summed input and weight to get the output value of the neuron. The difference between the target value and output value is the error and the partial derivative of the error is back propagated to adjust the weights in the layers and this iteration continues until the stopping condition reaches or the error is minimum. 
International Journal of Artificial Intelligence and Applications (IJAIA), Vol. 7, No. 5, September 2016

\section{Algorithm II: Backpropagation Training algorithm}

Input:

Initialize the weights to random values, stopping condition.

\section{Output:}

Trained network which has the output value with minimum error difference between actual and targeted output.

Method:

Step 1: While stopping condition are false, repeat Steps 2-9.

Step 2: For each training feature vector, repeat Steps 3-8.

\section{Feedforward Propagation}

Step 3: Every input neuron $\left(\mathrm{X}_{\mathrm{i}=1 \ldots \mathrm{n}}\right)$ receives input signal $\mathrm{X}_{\mathrm{i}}$ and broadcasts this to all units in the above layer (the hidden units).

Step 4: Each hidden neuron $\left(Z_{j=1 . p}\right)$ sums its weighted input signals,

$$
z_{-} i n_{j}=v_{o j}+\sum_{i} x_{i} v_{i j}
$$

applies its activation function (Sigmoid) to compute its output signal,

$$
z=f\left(z_{-} i n_{j}\right)
$$

and sends this signal to all units in the layer above (output units).

Step 5: Each output unit ( $\left.Y_{k}, k=1, \ldots, m\right)$ sums its weighted input signals,

$$
y_{-} i n_{k}=w_{o k}+\sum_{j} z_{j} w_{k j}
$$

and applies its activation function(sigmoid) to compute its output signal, the ouput (activation) of $Y_{\mathrm{k}}$ is denoted by $\mathrm{y}_{\mathrm{k}}$.

$$
y_{k}=f\left(y_{-} i n_{k}\right)
$$

\section{Backpropagation of error:}

Step 6: Each output unit ( $\mathrm{Y}_{\mathrm{k}=1 \ldots \mathrm{m}}$ ) receives a target pattern corresponding to the 
International Journal of Artificial Intelligence and Applications (IJAIA), Vol. 7, No. 5, September 2016

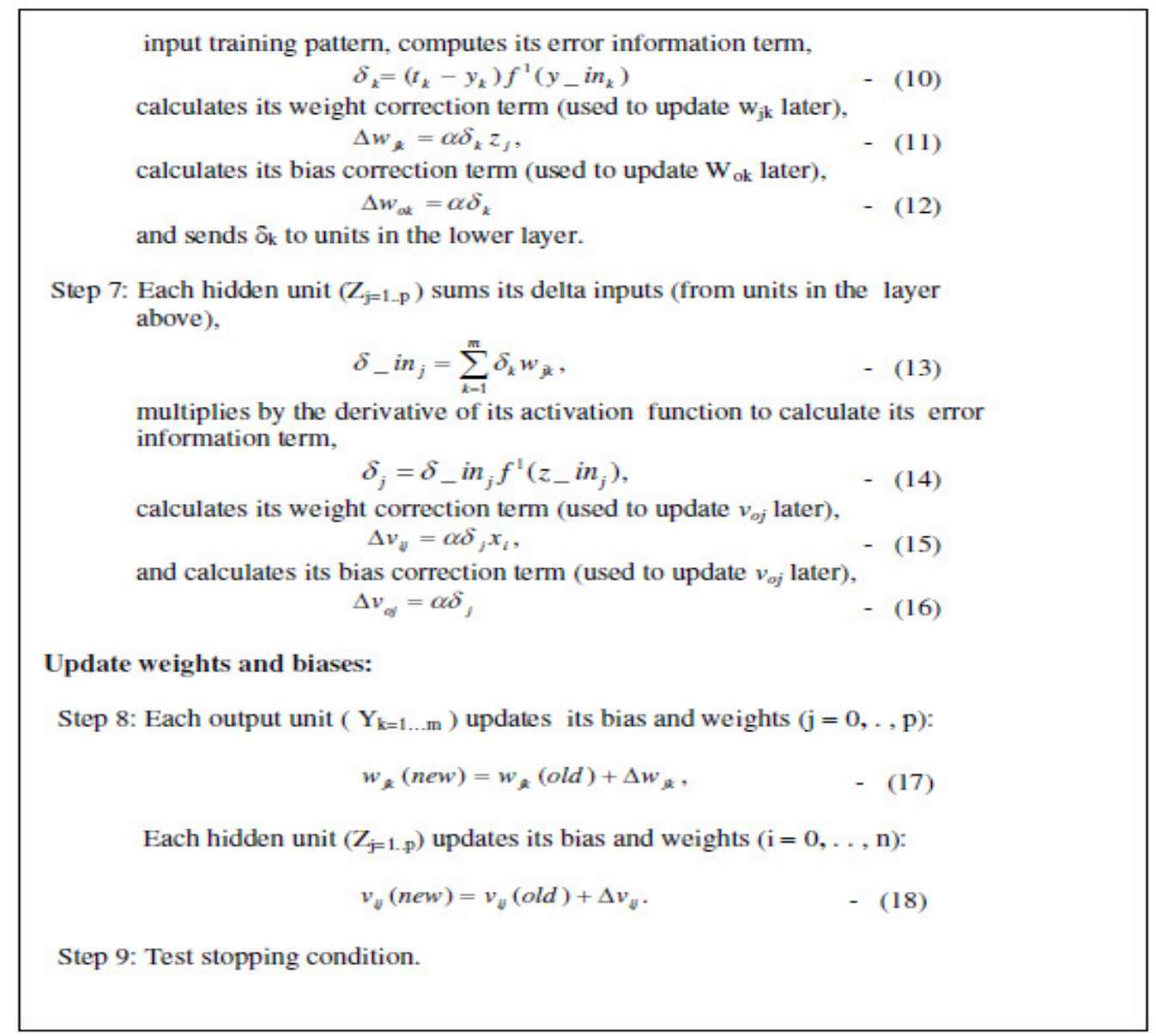

The update methodology for weights and bias values are different for output layer unit and hidden layer units. Each output unit receives a target pattern corresponding to the input training pattern. The weight and bias are updated by the error difference times the derivative of the output. In hidden units, the weights and bias are updated by multiplying the derivative of the activation function and the summed weights. All the updations are multiplied by learning rate, $\alpha$.

Levenberg-Marquart algorithm was designed to approach second order training speed without computing hessian matrix [24]. Hessian matrix can be approximated as $\mathbf{J}^{\mathrm{T}} \mathbf{J}$ and the gradient can be as $\mathrm{J}^{\mathrm{T}} \mathrm{e} . \mathrm{J}$ is the jacobian matrix and $\mathrm{e}$ is the vector of network errors. Trainlm algorithm minimizes the sum of the square error. Weight and bias performance Jocobian $J(\mathrm{x})$ and the errors $\mathrm{v}(\mathrm{x})$ are calculated.

The adjustments are calculated according to Levenberg-Marquart as,

$$
\Delta x=-\frac{J(x) v(x)}{J(x) J^{T}(x)+\mu_{K} I}
$$


International Journal of Artificial Intelligence and Applications (IJAIA), Vol. 7, No. 5, September 2016

the parameter $\mu_{K}$ in equation (19) is introduced in the hessian matrix of the trainlm algorithm and this parameter is of the reason for the best functioning and efficiency of the algorithm. It is responsible for the stability and speed of convergence of the algorithm. Weight adjustment is done by $\mathrm{W}+\Delta \mathrm{x}$ which is shown in the algorithm III.

\section{Algorithm III: Levenberg-Marguardt Algorithm}

\section{Input:}

Initial weight and bias values

\section{Output:}

Trained network which has the output value with minimum error difference between actual and targeted output.

Method:

Stepl:Compute the Jacobian, $\mathbf{J}$.

$$
J=\left[\begin{array}{ccc}
\frac{\partial F\left(x_{1}, w\right)}{\partial w_{1}} & \cdots & \frac{\partial F\left(x_{1}, w\right)}{\partial w_{W}} \\
\vdots & \ddots & \vdots \\
\frac{\partial F\left(x_{N}, w\right)}{\partial w_{1}} & \cdots & \frac{\partial F\left(x_{N}, w\right)}{\partial w_{W}}
\end{array}\right]
$$

Where $\mathrm{F}\left(\mathrm{x}_{\mathrm{i}}, \mathrm{w}\right)$ is the network function evaluated for the $i$-th input vector of the training set using the weight vector $\mathrm{w}$ and $\mathrm{w}_{\mathrm{j}}$ is the $j$-th element of the weight vector $\mathrm{w}$ of the network.

Step 2:Compute the error gradient $g=J^{t} \mathrm{E}$

Step 3:Approximate the Hessian using the cross product Jacobian as $\mathrm{H}=\mathrm{J}^{\mathbf{t}} \mathbf{J}$

Step 4 :Solve $(H+\lambda I) \delta=g$ to find $\delta$, where $\lambda$ is the damping factor, $\delta$ is the weight

update factor.

Step 5: Update the network weights $\mathbf{w}$ using $\boldsymbol{\delta}$

Step 6: Recalculate the sum of squared errors using the updated weights

Step 7: If the sum of squared errors has not decreased,

Discard the new weights, increase $\lambda$ using $\mathbf{v}$ and go to step 4 .

Step 8: Else decrease $\lambda$ using $\mathbf{v}$ and stop. 
International Journal of Artificial Intelligence and Applications (IJAIA), Vol. 7, No. 5, September 2016

\section{Experimental Results and Discussion}

Signature verification on bank cheques is a difficult task because of the limited number of samples available for training the neural network. In this modeling, three sets of signatures are taken and the features are extracted and used for training the neural network. $70 \%$ of the samples are used for training, $15 \%$ are used for validation and $15 \%$ are used for testing the samples.

Table I shows the neural network output values for three signature set. Each signature set contains 12 genuine signatures and 12 forged signatures. The table shows that the bold faced values are false positive values and there are no false negative values.

Table I. Neural Network Output values for three signature sets each of 24 (12 genuine and 12 forged)

\begin{tabular}{|c|c|c|c|}
\hline S.No. & Sign. Set 1 & Sign. Set 2 & Sign. Set 3 \\
\hline 1 & 1.208344 & 1.010238 & 0.711602 \\
\hline 2 & 1.048610 & 1.039663 & 0.709308 \\
\hline 3 & 1.027049 & 0.968979 & 0.565037 \\
\hline 4 & 1.662092 & 0.617007 & 0.406736 \\
\hline 5 & 1.015463 & 0.525320 & 1.348296 \\
\hline 6 & 1.520030 & 0.534797 & 0.551430 \\
\hline 7 & 0.998096 & 0.675068 & 0.379805 \\
\hline 8 & 1.295527 & 1.659889 & 0.907800 \\
\hline 9 & 1.075666 & 1.047988 & 0.964069 \\
\hline 10 & 0.977614 & 1.074687 & 0.932671 \\
\hline 11 & 1.678336 & 0.985079 & 0.462273 \\
\hline 12 & 0.126174 & 0.867442 & 0.672268 \\
\hline 13 & 0.020968 & -0.465160 & -0.995020 \\
\hline 14 & -0.066430 & 0.807860 & -0.555380 \\
\hline 15 & 0.317039 & 0.050329 & -0.168090 \\
\hline 16 & -0.244930 & 0.322038 & -0.777610 \\
\hline 17 & 0.206772 & 0.101294 & -0.439390 \\
\hline 18 & 0.050259 & 0.502559 & -0.158520 \\
\hline 19 & 0.129069 & -0.701200 & -0.099680 \\
\hline 20 & 0.207813 & 0.170739 & 0.785102 \\
\hline 21 & 0.079772 & -0.528180 & 0.584238 \\
\hline 22 & -0.352780 & 0.111966 & 0.043753 \\
\hline 23 & -0.448120 & -0.384800 & 0.876172 \\
\hline 24 & -0.457590 & 0.022204 & -0.534210 \\
\hline
\end{tabular}


Table II shows the TP, which indicates number of correct positive predictions, TN which indicates number of correct negatives, FP which indicates number of incorrect positives and FN which indicates number of incorrect positives. Each Signature set has 12 original signatures and 12 forged signatures.

Table II : Recognition Possibilities

\begin{tabular}{|c|c|c|c|}
\hline & Sign. Set 1 & Sign. Set 2 & Sign. Set3 \\
\hline TP & 11 & 12 & 10 \\
\hline TN & 12 & 12 & 12 \\
\hline FP & 1 & 0 & 2 \\
\hline FN & 0 & 0 & 0 \\
\hline
\end{tabular}

Sign.Set. 2 in table II shows that all the 24 signatures are identified correctly. Sign.Set. 1 has one identified one genuine signature as forged and Sign.Set.3 has identified two genuine signatures as forged.

Table III : Performance Measures in Percentages (\%)

\begin{tabular}{|l|c|c|c|c|}
\hline & $\begin{array}{c}\text { Sign. } \\
\text { Set 1 }\end{array}$ & $\begin{array}{c}\text { Sign. } \\
\text { Set 2 }\end{array}$ & $\begin{array}{c}\text { Sign. } \\
\text { Set 3 }\end{array}$ & Average \\
\hline Sensitivity & 100.00 & 100.00 & 100.00 & 100.00 \\
\hline Specificity & 92.307 & 100.00 & 85.714 & 95.673 \\
\hline Accuracy & 95.833 & 100.00 & 91.666 & 95.833 \\
\hline
\end{tabular}

Table III shows the performance measures of the proposed verification system. Sensitivity indicates the correct positive recognition and Specificity measures the negatively recognized samples. The sensitivity of the entire three signature sets are $100 \%$ and the specificity of signature set 2 is $100 \%$ shows that the system is viable for the signature verification. 
International Journal of Artificial Intelligence and Applications (IJAIA), Vol. 7, No. 5, September 2016

Table IV: Feature Vector Comparative Table

\begin{tabular}{|l|c|}
\hline Feature Vector & Accuracy \% \\
\hline Zernike and Wavelet Transforms [12] & 94.26 \\
\hline Zernike Moments [11] & 95.55 \\
\hline Surroundedness [4] & 91.67 \\
\hline $\begin{array}{l}\text { Zernike Moments and Radon } \\
\text { Tranformation [6] }\end{array}$ & 71.00 \\
\hline Zernike Moments and Circularity features & $\begin{array}{c}95.83 \\
\text { (Proposed) }\end{array}$ \\
\hline
\end{tabular}

Table IV shows the accuracy percentage for various features in combination with Zernike moments. When it is combined with the circularity and aspect ratio, the accuracy has increased in the proposed model. The sensitivity of all the three signature sets are $100 \%$ and the specificity of signature set 2 is $100 \%$ as shown in Figure.3. The overall accuracy of the system is $95 \%$ and FN rate is reduced when Zernike moments are used as feature with circularity and aspect ratio. Zernike moments are global shape descriptors [25].

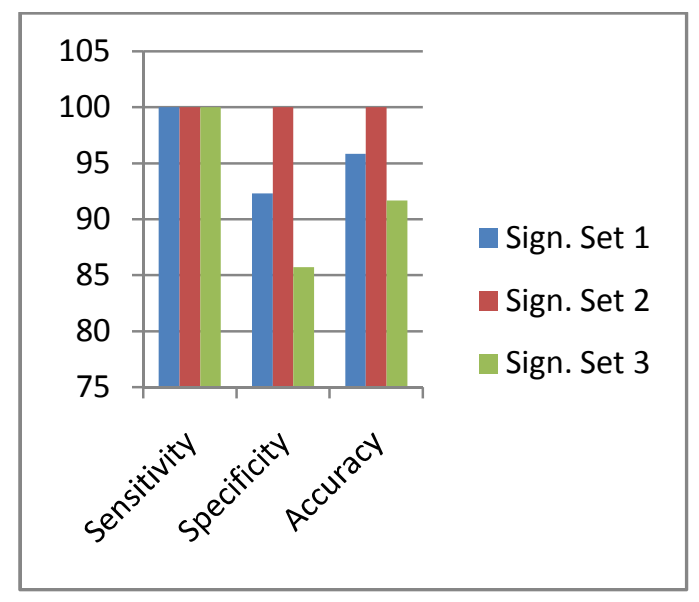

Figure. 3: Chart showing the performance Measures

ROC (Receiver Operating Characteristic curve) is another performance measure of a classifier. ROC curve represents how FAR varies with respect to FRR. ROC shows the tradeoff between sensitivity and specificity. The curve goes close to the left hand border and top if the classification is accurate [26]. Figure 4.a,b and c shows the ROC curves for the proposed system for the three different signature sets. It is a plot of true positive against the false positive rates. 
International Journal of Artificial Intelligence and Applications (IJAIA), Vol. 7, No. 5, September 2016

True positive rate is the sensitivity. False positive rate is also known as fall-out which is calculated as (1- specificity).

Table IV: Sensitivity and Specificity values for different thresholds for ROC curve

\begin{tabular}{|c|c|c|c|c|c|c|c|c|c|}
\hline & \multicolumn{3}{|c|}{ When threshold =8 } & \multicolumn{3}{c|}{ When threshold =7 } & \multicolumn{3}{c|}{ When threshold =9 } \\
\cline { 2 - 10 } & $\begin{array}{c}\text { Sign. } \\
\text { Set 1 }\end{array}$ & $\begin{array}{c}\text { Sign. } \\
\text { Set 2 }\end{array}$ & $\begin{array}{c}\text { Sign. } \\
\text { Set 3 }\end{array}$ & $\begin{array}{c}\text { Sign. } \\
\text { Set 1 }\end{array}$ & $\begin{array}{c}\text { Sign. } \\
\text { Set 2 }\end{array}$ & $\begin{array}{c}\text { Sign. } \\
\text { Set 3 }\end{array}$ & $\begin{array}{c}\text { Sign. } \\
\text { Set 1 }\end{array}$ & $\begin{array}{c}\text { Sign. } \\
\text { Set 2 }\end{array}$ & $\begin{array}{c}\text { Sign. } \\
\text { Set 3 }\end{array}$ \\
\hline Sensitivity & 1.0000 & 1.0000 & 1.0000 & 1.0000 & 0.9231 & 0.9167 & 1.0000 & 1.0000 & 1.0000 \\
\hline Specificity & 0.9231 & 1.0000 & 0.8571 & 0.9231 & 1.0000 & 0.9167 & 0.9232 & 0.8571 & 0.7500 \\
\hline $\mathbf{1 -}$ & 0.0769 & 0.0000 & 0.1429 & 0.0769 & 0.0000 & 0.0833 & 0.0769 & 0.1429 & 0.2500 \\
\hline
\end{tabular}

The ROC curves are drawn from the Table IV above. Three different threshold values are used to plot the ROC curve. The values are plotted with 1-specificity values along $\mathrm{x}$-axis and sensitivty in the $y$ axis.

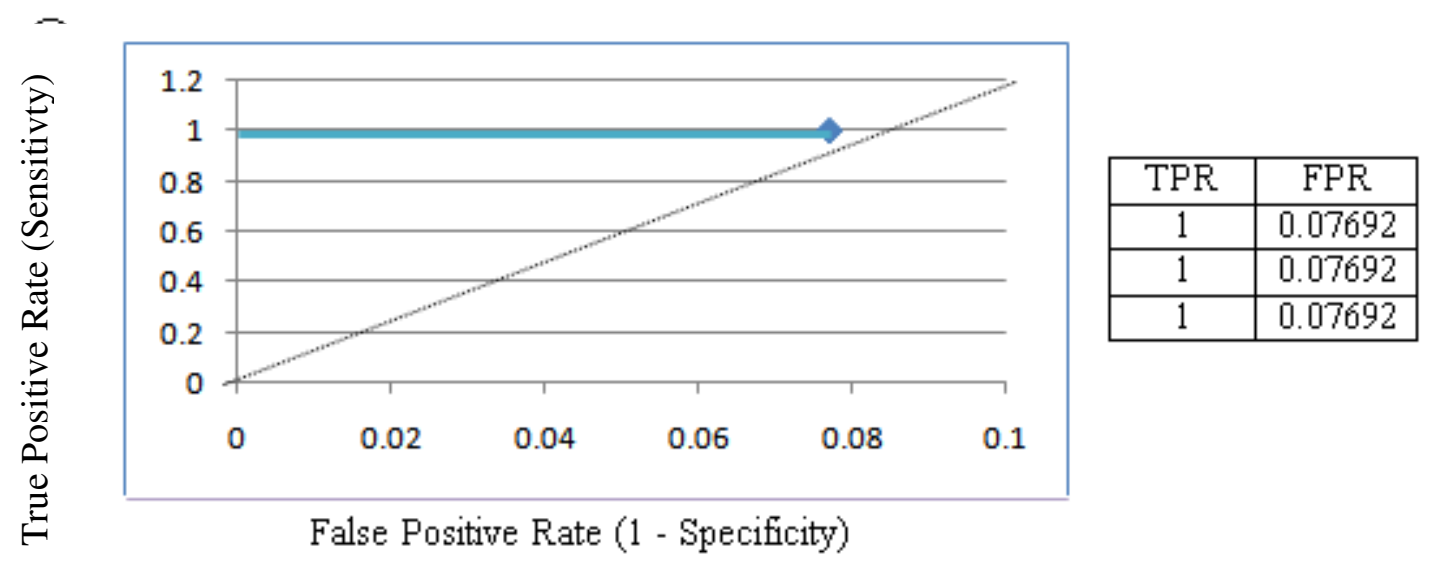

Figure 4.a: ROC Curve for Signature set 1

Figure 4.a shows the ROC plot for the signature set 1. Since the TPR and FPR (1, 0.07692) are constant for the 3 threshold values, a straight line above the diagonal is formed. They are used to improve the system performance further. If the curve comes close to the 45 degree dotted line, the test is less accurate. The curve fits good and shows that the classifier modeled is accurate. The curve is drawn for a set of signature with different threshold values. 
International Journal of Artificial Intelligence and Applications (IJAIA), Vol. 7, No. 5, September 2016

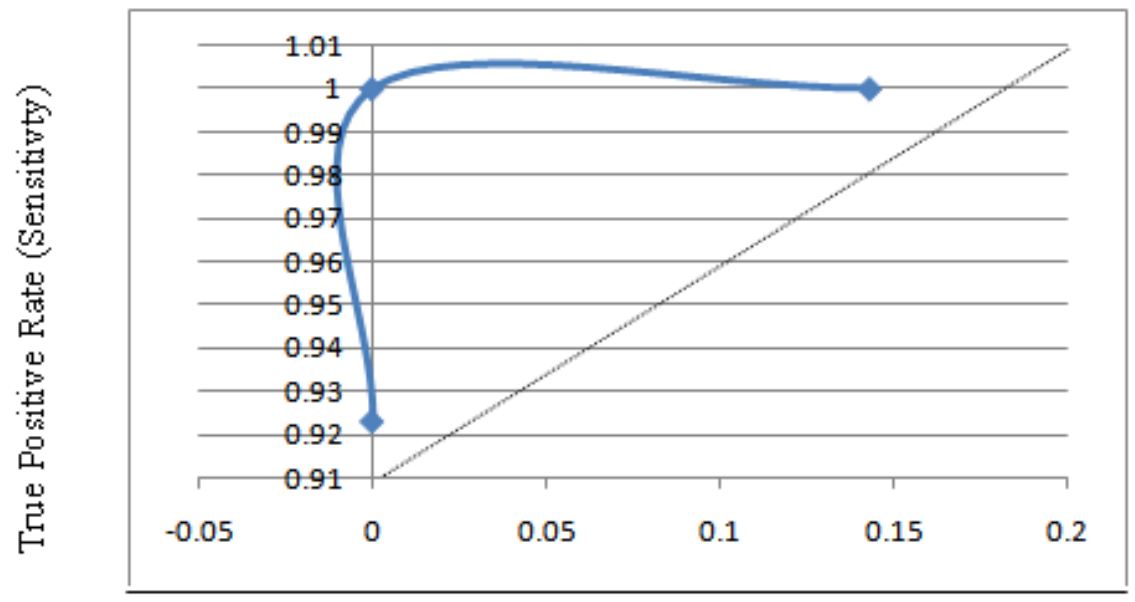

False Positive Rate (1 - Specificity)

\begin{tabular}{|c|c|}
\hline TPR & FPR \\
\hline 0.92308 & 0.00000 \\
\hline 1.00000 & 0.00000 \\
\hline 1.00000 & 0.14286 \\
\hline
\end{tabular}

Figure 4.b: ROC Curve for Signature set 2

Figure 4.b shows the ROC curve for the signature set 2. It is plotted for values of FPR ( 0.0, 0.0, $0.14286)$ against the TPR $(09.2308,1,1)$. The curve covers a maximum area in the plot above the diagonal, which shows that the accuracy is also higher.

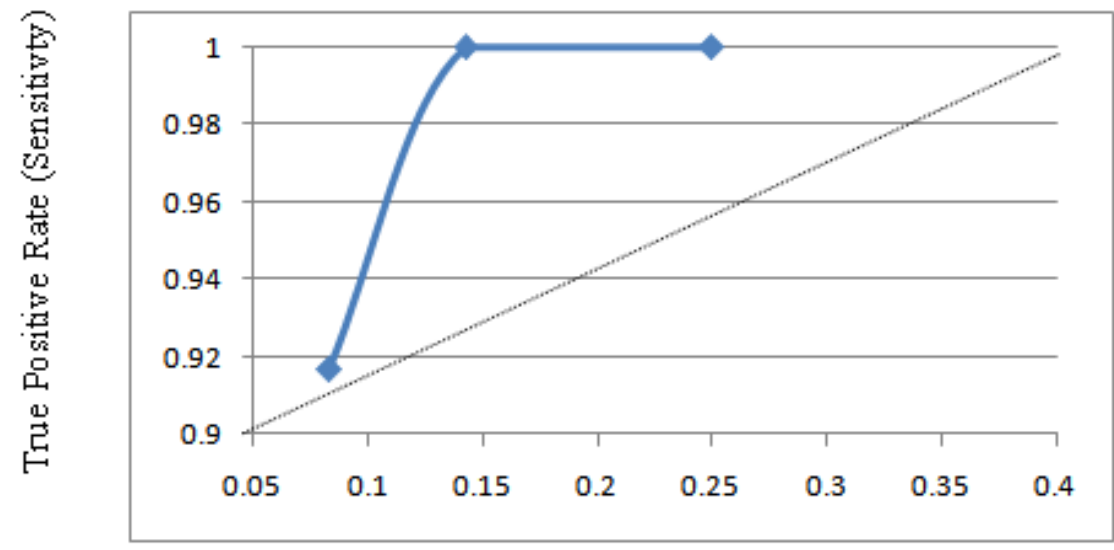

\begin{tabular}{|c|c|}
\hline TPR & FPR \\
\hline 0.91667 & 0.08333 \\
\hline 1.00000 & 0.14286 \\
\hline 1.00000 & 0.25000 \\
\hline
\end{tabular}

False Positive Rate (1 - Specificity)

Figure 4.c: ROC Curve for Signature set 3 
Figure 4.c shows the ROC plot for the signature set 3. FPR values ( $0.08333,0.14286,0.2500)$ are plotted against the TPR values $(0.91667,1,1)$. The curve covers more area above the dotted diagonal line which indicates that the designed classifier is good. If the area under the curve is maximum, the accuracy is higher. Any curve falling diagonal or below the diagonal, the classification is less accurate [27]. AUC (Area under curve) of ROC is also a measure of accuracy. Classifier with area lesser than 0.5 is considered less accurate.The area of a square is 1 and that of triangle is 0.5 . The accuracy of the classifier is increasing by its curve area. The right lower part of the graph forms a triangle below the dotted line and the area is 0.5 In the above ROC curves, the area of all the three signature set ROC are greater than 0.5 so the modeled classifier yields good results.

\section{CONCLUSION}

Offline signature verification is the most easiest and non-invasive biometric method. Signatures can be identified by their geometrical shape. The system modeled is generous to intra-personnel signature variations and rude to inter-personnel signature variation ones, the forgery. Zernike moments itself enhances signature verification because of their scale and rotation invariancy. When it is combined with features like circularity and aspect ratio, it proved to yield more accuracy than the state of the art results. These feature vectors when combined together, gives an accuracy of $95.83 \%$ which is better than the combination with other feature vectors. The ROC curve is plotted to measure the performance and shows good accuracy results.

\section{REFERENCES}

[1] Indrajit Bhattacharya, Prabir Ghosh and Swarp Biswas, Offline Signature Verification Using Pixel Matching Technique, Elsevier Procedia Technology, vol. 10, pp. 970-977, 2013.

[2] Ashok Kumar.D and Dhandapani.S, A Bank Cheque Signature Verification System using FFBP Neural Network Architecture and Feature Extraction based on GLCM, IJETTCS, vol. 3, issue 3, pp. 46-52, June 2014.

[3] Saki. F, A. Tahmasbi.A, Soltanian-Zadeh.H, Shokouhi.S.B, Fast opposite weight learning rules with application in breast cancer diagnosis, Comput. Biol. Med., vol. 43, no. 1, pp. 32-41, 2013.

[4] Rajesh Kumar, J D Sharma, Bhabatosh Chanda, Writer-Independent Off-line Signature Verification using Surroundedness Feature, Pattern Recognition Letters October 10, 2011.

[5] Hai Lin and Hai-Zhou Li, Chinese signature verification with moment invariants, IEEE International Conference on Systems, Man, and Cybernetics, Beijing, vol.4., pp. 2963-2968, 1996.

[6] Pushpalatha K. N., Kumar Gautham. A, Shashikumar. R, Offline Signature Verification with Random and Skilled Forgery Detection Using Polar Domain Features and Multi Stage ClassificationRegression Model, International Journal of Advanced Science and Technology, vol.59, pp. 27-40, 2013.

[7] Khotanzad.A and Hong.Y.H., Invariant Image Recognition by Zernike Moments, IEEE Transactions on Pattern Analysis and Machine Intelligence archive vol. 12, issue 5, pp. 489-497, May 1990.

[8] Sohail Jafar and Rashid Jalal Qureshi, Off-line signature verification using structural features, 7th International Conference on Frontiers of Information Technology, Abbottabad, Pakistan, December 16-18, 2009.

[9] Jovisa Zunic and Kaoru hirota, Measuring Shape Circularity, Proceedings of the 13th Iberoamerican congress on Pattern Recognition: Progress in Pattern Recognition, Image Analysis and Applications, pp. 94 - 101, CIARP, 2008. 
International Journal of Artificial Intelligence and Applications (IJAIA), Vol. 7, No. 5, September 2016

[10] Huazhong Shu, Limin Luo and Jean-Louis, Moment based Approaches in Imaging, IEEE Eng Med Biol Magazine, vol.26(5), pp. 70-74, Sep-Oct, 2007.

[11] Divjyot Singh Puri and Maitreyee Dutta. Article: A Comparative Analysis of Offline Signature Verification using Zernike Moment and Minutiae using Artificial Neural Network Approach. International Journal of Computer Applications 101(14):13-19, September 2014.

[12] Neo Han Foon, Ying-Han Pang and D.N.C.Ling, An efficient method for human face recognition using wavelet transform and Zernike moments, International Conference on Computer Graphics, Imaging and Visualization, pp. 65-69, CGIV, July 2004.

[13] Pham, Hong-Ha Le, Nang-Toan Do, Offline Handwritten Signature Verification using Local and Global Features, Springer Annals of Mathematics and Artificial Intelligence, vol 75 , issue 1, pp. 231-247, October 2015.

[14] Cemil Oz, Signature Recognition and Verification with Artificial Neural Network Using Moment Invariant Method, Springer, Advances in Neural networks, vol. 3497 of the series Lecture Notes in Computer Science, pp. 195-202, 2005.

[15] Pradeep Narayanan Narwade, S.V.Bonde and D.D. Doye, Offline Signature Verification using Shape Dissimilarities, IEEE International Conference on Communication, Information and Computing Technology, pp. 1-6, Jan 2015.

[16] Moises Diaz, Miguel A Ferrer, George S Eskander and Robert Sabourin, Generation of Duplicated Off-line Signature Images for Verification Systems, IEEE Transactions on Pattern Analysis and Machine Intelligence, issue: 99, 2016.

[17] Jasmine etal, Bank Cheque Authentication using Signature, IJARCSSE, vol 3, issue 5, May 2013.

[18] Ashok Kumar.D and Dhandapani.S, A Novel Signature Verification System on Bank Cheque with Fractal Dimensions and Connected Components, IJAER, vol 10, No.(14), pp. 34383-34389, 2015.

[19] Alireza Khotanzad and Yaw Hua Hong ,Invariant Image Recognition by Zernike Moments, IEEE Transactions on Pattern Analysis and Machine Intelligence. vol. 12, No. 5, May 1990.

[20] Amanatiadis. A and et al, Evaluation of shape descriptors for shape-based image Retrieval, IET Image Process, vol. 5, issue. 5, pp. 493-499, 2011.

[21] A. Tahmasbi, F. Saki, S. B. Shokouhi, Classification of Benign and Malignant Masses Based on Zernike Moments, Comput. Biol. Med., vol. 41, no. 8, pp. 726-735, 2011.

[22] Nilesh Y. Choudhary, Mrs. Rupal Patil, Dr. Umesh. Bhadade, Prof. Bhupendra M Chaudhari, Signature Recognition \& Verification System Using Back Propagation Neural Network, IJIEASR ISSN: 2319-4413, vol. 2, No. 1, January 2013.

[23] Salavi Rashmi, Sohani Mandar, Textural Feature Based Image Classification Using Artificial Neural Network, Springer, volume 125 of the series Communications in Computer and Information Science pp. 62-69, 2011.

[24] Ozgur Kisi and Erdal Uncuoglu, Comparision of three back-propagation training algorithms for two case studies, Indian Jounal of Engineering and Material Sciences, vol 12, pp. 434-442, Oct, 2005.

[25] Harman Preet Kair and Anmol Aharma, Offline Handwritten Signature Verification using Zernike Moments, NCVPRIPG, pp. 1-4, Dec, 2015.

[26] Luiz S Oliveria and Edson Justino, Combining Classifiers in the ROC -space for Offline Signature Verification, JUCS, vol 14(2), pp. 237-251, Jan, 2008.

[27] Srivastava.K, Subhash Chandra and Sushila V Maheshkar, Offline Signature Verification with ANN using Shape Properties a Feasibility Report, ACSIT, vol 2(3), pp. 267-271, Mar,2015. 
International Journal of Artificial Intelligence and Applications (IJAIA), Vol. 7, No. 5, September 2016

\section{AUTHORS}

Dr. D. Ashok Kumar did his Master Degree in Mathematics and Computer Applications in 1995 and completed Ph.D., on Intelligent Partitional Clustering Algorithm's in 2008, from Gandhigram Rural Institute Deemed University, Gandhigram, Tamilnadu, INDIA. He is currently working as Associate Professor and Head in the Department of Computer Science and Applications, Government Arts College, Tiruchirapalli- 620 022, Tamilnadu, INDIA. His research interest includes Pattern Recognition and Data Mining by various soft computing approaches viz., Neural Networks, Genetic Algorithms, Fuzzy Logic, Rough set, etc., Cell: +91-9443654052.

S.Dhandapani completed his M. C. A. Degree from Bharathiar University, Coimbatore, TamilNadu and M.Phil. from Periyar University, Salem. He is currently a Ph.D. research scholar in the field of Pattern Recognition and Image Processing at Bharathidasan University, Tiruchirapalli, Tamil Nadu. Cell: +91-9943357265.
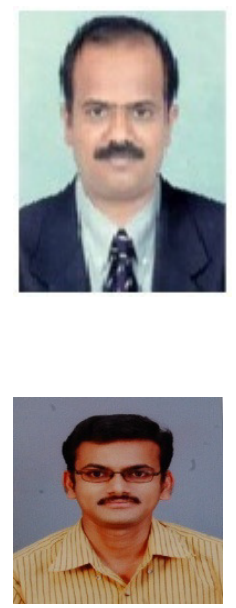